\title{
Weak evidence suggests higher risk for bracket bonding failure with self-etch primer compared to conventional acid etch over 12 months
}

\author{
Abstracted from \\ Fleming PS, Johal A, Pandis N. \\ Self-etch primers and conventional acid-etch technique for orthodontic bonding: \\ a systematic review and meta-analysis. Am J Orthod Dentofacial Orthop. 2012; 142: 83-94. \\ Address for correspondence: Padhraig S. Fleming, Locum Consultant, \\ Barts and The London NHS Trust, Dental Institute, Whitechapel, \\ London E1 1BB, United Kingdom. E-mail: padhraig.fleming@gmail.com.
}

\section{Question: When bonding orthodontic appliances are 1-step bonding approaches more effective than 2-step approaches?}

Data sources Medline, Embase, Cochrane Oral Health Group's Trials Register and the Cochrane Central Register of Controlled Trials (CENTRAL). Unpublished data were sought by searching ClinicalTrials. gov, the National Research Register and Pro-Quest Dissertation Abstracts and Thesis database. There were no language restrictions. Study selection Randomised and controlled clinical trials (including split mouth) directly comparing self-etch and acid-etch primers including patients with full-arch, fixed and bonded orthodontic appliances (not banded) with follow-up periods of at least 12 months were included.

Data extraction and synthesis Two authors abstracted data independently, with disagreements being resolved by a third. The Cochrane Risk of Bias tool was used to assess study quality. A random effects meta-analysis was undertaken.

Results Eleven studies were included in the qualitative summary with five studies contributing to a meta-analysis. These five studies ( $\mathrm{n}=3444$ brackets, 1721 acid-etch, 1723 self-etch) had relatively low statistical and clinical heterogeneity. Meta-analysis demonstrated a tendency for a higher risk of failure (odds ratio $1.35 ; 95 \% \mathrm{Cl}, 0.99$ $1.83 ;$ P 5 0.06) with self-etch primers. The use of self-etch techniques was also associated with a small but statistically significant time saving (weighted mean difference 23.2 seconds per bracket; $95 \% \mathrm{Cl}$, 20.7$25.8 ; P \backslash 0.001)$. There was insufficient evidence to assess the effect of bonding modality on demineralisation rates.

Conclusions There is weak evidence indicating higher odds of failure with self-etch primer than acid-etch over 12 months in orthodontic patients, and there is strong evidence that a self-etch primer is likely to result in modest time savings (eight minutes for full bonding) compared with acid-etch.

\section{Commentary}

Dental adhesive systems are the cornerstone for bonding orthodontic brackets. While acid-etch (AE) technique is commonly used, the pursuit of faster and simpler procedures has led to development of self-etch adhesives which are classified according to the number of application steps into one step or two steps. One step self-etch primer (SEP) is further classified according to the number of components into two component adhesives (eg Transbond Plus, Clearfil SE) and one component adhesives (eg Ideal 1, iBond). ${ }^{1,2}$ The aim of this systematic review was to compare AE and SEP with respect to attachment failure rate and time taken to place attachments.

Two electronic databases and four trial registries were searched through July 2011 with no language restriction. A thorough search of unpublished literature was performed as well. Two investigators independently assessed research for inclusion in the review, assessed risk of bias and extracted data. Any disputes were resolved by discussion and consultation with a third author.

Meta-analysis was performed for two outcomes; bracket failure and required time to bond. Meta-analysis of bracket failure included five RCTs with 1724 and 1721 brackets in SEP (Transbond Plus) and $\mathrm{AE}$ groups respectively, while information for the time required to bond was found in two trials. One included study (Aljubouri et al. ${ }^{3}$ ) observed bond failure rate in paired brackets (353 AE and 353 SEP), while the authors mistakenly included the whole set of brackets (388 AE and $380 \mathrm{SEP}$ ). Although the difference is small, it might have an impact on statistical significance of bond failure risk (odds ratio=1.35; 95\% CI, 0.99-1.83; P=0.06)

Two dilemmas faced the authors in quantitative analysis; clustering effect ${ }^{4}$ and the exclusion of House et al. study. ${ }^{5}$ Regarding clustering effect, neither a measure of correlation of data nor entire data sets were reported in split mouth RCTs. Hence, the authors depended only on cluster correlation which might have altered the results towards the null. The second dilemma was the exclusion of House et al. trial which ended prematurely due to an excessive number of failures in SEP (Ideal 1) arm. To overcome this problem, a sensitivity analysis including this trial was performed. The direction of the results remained the same.

Quality of reporting trials was assessed using Cochrane Collaboration Risk of Bias tool. Trials with high risk of bias were excluded from meta-analysis. Four split mouth RCTs discussing bond failure risk were excluded due to inadequate or unclear randomisation procedures. These four trials included 1458 brackets in the SEP group and 1460 brackets in the AE group. No sensitivity 
analysis including trials with high risk of bias was performed; hence their effect on the direction of the results cannot be concluded.

\section{Ahmed ElKhadem ${ }^{1}$ and Noha Orabi ${ }^{2}$ \\ ${ }^{1}$ Evidence Based Dentistry Centre, Faculty of Oral and Dental Medicine, Cairo University. \\ 2 Operative Dentistry Department, Faculty of Oral and Dental Medicine, Cairo University, Egypt}

1. Lamper T, Ilie N, Huth KC, Rudzki I, Wichelhaus A, Paschos E. Self-etch adhesives for the bonding of orthodontic brackets: faster, stronger, safer? Clin Oral Investig. 2013. [Epub ahead of print]

2. Isman E, Karaarslan ES, Oksayan R, Tuncdemir AR, Usumez S, Adanir N, et al. Inadequate shear bond strengths of self-etch, self-adhesive systems for secure orthodontic bonding. Dent Mater J. 2012; 31: 947-953.

3. Aljubouri YD, Millett DT, Gilmour WH. Six and 12 months' evaluation of a self-etching primer versus two-stage etch and prime for orthodontic bonding: a randomized clinical trial. Eur J Orthod. 2004; 26: 565-571.

\section{Practice points}

- Weak evidence favours AE over SEP technique regarding bonding failure risk. Hence, the choice of bonding technique is still dependent on operator selection.

- On dual arch bracket placement, SEP saves up to eight minutes of chair side time.

4. Pandis N, Walsh T, Polychronopoulou A, Katsaros C, Eliades T. Split-mouth designs in orthodontics: an overview with applications to orthodontic clinical trials. Eur / Orthod. 2013. [Epub ahead of print]

5. House K, Ireland AJ, Sherriff M. An investigation into the use of a single component self-etching primer adhesive system for orthodontic bonding: a randomized controlled clinical trial. J Orthod. 2006; 33: 38-44.

Evidence-Based Dentistry (2013) 14, 52-53. doi:10.1038/sj.ebd.6400936 